\title{
Control Mechanism of Aeroelastic System based on Piezoelectric Actuator
}

\author{
Jie Sun ${ }^{\mathrm{a}}$ and Min $\mathrm{Li}^{\mathrm{b}}$ \\ School of Aeronautic Science and Engineering, Beijing University of Aeronautics and \\ Astronautics, Beijing 100191, China \\ asunjie1101@126.com, blimin@buaa.edu.cn
}

\begin{abstract}
Keywords: aeroelasticity; MFC; negative velocity feedback; additional aerodynamic force; control mechanism.

Abstract. Mechanism of aeroelastic control is more complicated than that of vibration control based on piezoelectric material, and analyzing the control mechanism is of great significance for the research of aeroelastic control. The macro fiber composite (MFC) actuator is modeled by the load simulation method. The control law of the aeroelastic model is designed by the negative velocity feedback algorithm. The effects of the additional aerodynamic force and the piezoelectric driving force on the aeroelastic control system are studied, and the control mechanisms of the system are analyzed. The results show that the additional aerodynamic force can improve damping behavior of the aeroelastic open-loop system below the flutter velocity. With the increase of airflow velocity, the damping effect of the additional aerodynamic force on the aeroelastic closed-loop system is increasingly strong, but that of the piezoelectric driving force on the system becomes gradually weak. In aeroelastic control, the energy consumption of the system is mainly dependent on the additional aerodynamic force, which is obviously different from the control mechanism that in vibration control.
\end{abstract}

\section{Introduction}

The aeroelastic effect has an important influence on the safety and performance of the aircraft. In recent years, intelligent materials, especially piezoelectric materials, have been widely used in the aircraft aeroelastic control due to their excellent driving characteristics and high energy density.

The dynamic aeroelastic control based on piezoelectric actuator is the most common, such as active flutter suppression ${ }^{[1,2]}$ and buffeting active control ${ }^{[3]}$, in which satisfactory control effects are obtained. At present, great progress has been made for active vibration control using piezoelectric materials ${ }^{[4]}$, however, the research of aeroelastic control using piezoelectric materials is far behind the former. In particular, the study on the mechanism of the piezoelectric actuator in the aeroelastic control is less. The influence of the additional aerodynamic force and the piezoelectric driving force on the aeroelastic control is not clear, and related analysis methods are also relatively scarce. Therefore, studying the mechanism of aeroelastic control is of great significance for the research of aeroelastic control.

In this paper, taking dynamic aeroelastic response control of a cantilever plate as the typical aeroelastic control. The mechanism of aeroelastic control is analyzed, and the control mechanism is explained from the aspect of energy consumption, so as to provide a useful reference for the study of aeroelastic control problems.

\section{Aeroelastic model and control law design}

The structural dynamics of the cantilever plate using piezoelectric actuators can be written as

$$
\boldsymbol{M}_{\mathrm{uu}} \ddot{\boldsymbol{u}}+\boldsymbol{C}_{\mathrm{uu}} \dot{\boldsymbol{u}}+\boldsymbol{K}_{\mathrm{uu}} \boldsymbol{u}=\boldsymbol{F}-\boldsymbol{K}_{\mathrm{u \varphi}} \boldsymbol{U} .
$$

where $\boldsymbol{u}$ is the displacement, $\boldsymbol{F}$ represents the external force, $\boldsymbol{M}_{\mathrm{uu}}$ is the structural mass matrix, $\boldsymbol{C}_{\mathrm{uu}}$ is the damping matrix, $\boldsymbol{K}_{\mathrm{uu}}$ is the stiffness matrix, $\boldsymbol{K}_{\mathrm{u} \varphi}$ denotes the piezoelectric coupling matrix. The last term in the right-hand side of Eq. (1) $\boldsymbol{K}_{\mathrm{u \varphi}} \boldsymbol{U}$ can be calculated by the load simulation method ${ }^{[5]}$. 
Introducing the modal matrix $\Phi$, then, Eq. (1) can be expressed as

$$
\overline{\boldsymbol{M}}_{\mathrm{uu}} \ddot{\boldsymbol{q}}+\overline{\boldsymbol{C}}_{\mathrm{uu}} \dot{\boldsymbol{q}}+\overline{\boldsymbol{K}}_{\mathrm{uu}} \boldsymbol{q}=\overline{\boldsymbol{F}}-\overline{\boldsymbol{F}}_{\mathrm{q}} .
$$

where

$$
\begin{aligned}
& \overline{\boldsymbol{M}}_{\mathrm{uu}}=\boldsymbol{\Phi}^{\mathrm{T}} \boldsymbol{M}_{\mathrm{uu}} \boldsymbol{\Phi}, \overline{\boldsymbol{C}}_{\mathrm{uu}}=\boldsymbol{\Phi}^{\mathrm{T}} \boldsymbol{C}_{\mathrm{uu}} \boldsymbol{\Phi}, \overline{\boldsymbol{K}}_{\mathrm{uu}}=\boldsymbol{\Phi}^{\mathrm{T}} \boldsymbol{K}_{\mathrm{uu}} \boldsymbol{\Phi} . \\
& \overline{\boldsymbol{F}}=\boldsymbol{\Phi}^{\mathrm{T}} \boldsymbol{F}=\overline{\boldsymbol{F}}_{\mathrm{as}}+\overline{\boldsymbol{F}}_{\mathrm{bs}}, \overline{\boldsymbol{F}}_{\mathrm{q}}=\boldsymbol{\Phi}^{\mathrm{T}} \boldsymbol{K}_{\mathrm{u} \varphi} \boldsymbol{U} .
\end{aligned}
$$

where $\boldsymbol{q}$ is the modal coordinate, $\overline{\boldsymbol{F}}_{\mathrm{as}}$ is generalized aerodynamic force vector, $\overline{\boldsymbol{F}}_{\mathrm{bs}}$ is generalized external force. The unsteady aerodynamic forces on the cantilever plate can be calculated by the doublet-lattice method $^{[6]}$. The detailed derivation process of the aeroelastic state-space equation can refer to the literature ${ }^{[7]}$.

So the aeroelastic state-space equation of the cantilever plate can be written as

$$
\begin{aligned}
& \left\{\begin{array}{l}
\dot{\boldsymbol{X}}=\boldsymbol{A}_{\mathrm{m}} \boldsymbol{X}+\boldsymbol{B}_{\mathrm{m}} \boldsymbol{U}+\boldsymbol{D}_{\mathrm{m}} \overline{\boldsymbol{F}}_{\mathrm{bs}} \\
\boldsymbol{Y}=\boldsymbol{C}_{\mathrm{m}} \boldsymbol{X}
\end{array}\right. \\
& \boldsymbol{A}_{\mathrm{m}}=\left[\begin{array}{ccc}
0 & \boldsymbol{I} & 0 \\
-\widehat{\boldsymbol{M}}_{\mathrm{uu}}^{-1} \widehat{\boldsymbol{K}}_{\mathrm{uu}} & -\widehat{\boldsymbol{M}}_{\mathrm{uu}}^{-1} \widehat{\boldsymbol{C}}_{\mathrm{uu}} & q_{\mathrm{d}} \widehat{\boldsymbol{M}}_{\mathrm{uu}}^{-1} \boldsymbol{D}_{\mathrm{s}} \\
0 & \boldsymbol{E}_{\mathrm{s}} & \left(\frac{V}{b}\right) \boldsymbol{R}_{\mathrm{s}}
\end{array}\right], \boldsymbol{B}_{\mathrm{m}}=\left[\begin{array}{c}
0 \\
-\widehat{\boldsymbol{M}}_{\mathrm{uu}}^{-1} \boldsymbol{\Phi}^{\mathrm{T}} \boldsymbol{K}_{\mathrm{u} \varphi} \\
0
\end{array}\right], \boldsymbol{D}_{\mathrm{m}}=\left[\begin{array}{c}
0 \\
\boldsymbol{M}_{\mathrm{uu}}^{-1} \\
0
\end{array}\right], \boldsymbol{X}=\left[\begin{array}{c}
\boldsymbol{q} \\
\dot{\boldsymbol{q}} \\
\boldsymbol{X}_{\mathrm{a}}
\end{array}\right] . \\
& \boldsymbol{C}_{\mathrm{m}}=\left[\begin{array}{llll}
0 & \boldsymbol{\psi}_{h}^{\mathrm{T}} & 0
\end{array}\right], \boldsymbol{\psi}_{h}^{\mathrm{T}}=\left[\begin{array}{llll}
T_{z h}^{1} & T_{z h}^{2} & \cdots & T_{z h}^{m}
\end{array}\right] .
\end{aligned}
$$

where $\boldsymbol{X}_{\mathrm{a}}$ is the aerodynamic state variable, $\widehat{\boldsymbol{M}}_{\mathrm{uu}}, \widehat{\boldsymbol{C}}_{\mathrm{uu}}$ and $\widehat{\boldsymbol{K}}_{\mathrm{uu}}$ are respectively the aerodynamic mass matrix, aerodynamic damping matrix and aerodynamic stiffness matrix, $\boldsymbol{D}_{s}, \boldsymbol{E}_{s}$, and $\boldsymbol{R}_{s}$ are parameters matrix, $b$ is the reference half-chord length, $V$ is the airflow speed, and $T_{z h}^{m}$ represents the displacement in the $z$ direction of point $h$ in the $m$-order mode.

In this paper, the control law of the aeroelastic model is designed by using the algorithm of velocity negative feedback. The velocity negative feedback can be expressed as

$$
\boldsymbol{U}=-\boldsymbol{K}_{\mathrm{f}} \boldsymbol{Y}=-\boldsymbol{K}_{\mathrm{f}} \boldsymbol{C}_{\mathrm{m}} \boldsymbol{X} .
$$

where $\boldsymbol{K}_{\mathrm{f}}$ represents feedback gain. In this paper, the maximum driving voltage of MFC is $1000 \mathrm{~V}$.

Substituting Eq. (8) into Eq. (5), the aeroelastic state-space equation of the cantilever plate structure with the velocity negative feedback control can be obtained.

\section{Numerical simulation and discussions}

Response control of a rectangular cantilever plate under free vibration is analyzed as the typical case of the aeroelastic control. The finite element model is established by using MSC.Nastran, as shown in Fig.1. The geometrical and material parameters of the plate are respectively as follows: $A B=C D=400 \mathrm{~mm}, A D=B C=200 \mathrm{~mm}$, the thickness of the plate is $2.15 \mathrm{~mm}$, Young's modulus of elasticity is 70GPa, Poisson's ratio is 0.3 , and the density is $2700 \mathrm{~kg} / \mathrm{m}^{3}$. The damping of the plate is not considered here. Our main consideration here is the control mechanism of the aeroelastic system, as for the issues like optimal location of the piezoelectric actuators will not be considered. One MFC patch is bonded near the plate root and the direction of the piezoelectric fiber is same with the 
direction of $y$ axis. The thickness of the MFC is $0.3 \mathrm{~mm}$ and the parameters of the MFC actuator are shown in Table 1.The first five truncated modal frequencies of the plate are given in Table 2. Point $E$ is selected as the sensing point and point $C$ the response output point.

Open-loop and closed-loop displacement responses and control effects of the aeroelastic model. Because the vibration energy of the cantilever plate is mainly concentrated in the first bending mode, $\boldsymbol{q}=(1,0.15,0,0,0)^{\mathrm{T}}$ is selected as the initial condition of the free vibration. The first bending modal response is mainly controlled by the aeroelastic control model.

In order to quantify the displacement response and control effect of the aeroelastic model, define percentage reduction as follows: $R=(1-\bar{B} / \bar{A}) \times 100 \%$. Where, $\bar{B}$ expresses root mean square (RMS) value of closed-loop displacement response of the output point under different airflow velocities, $\bar{A}$ expresses RMS value of corresponding open-loop displacement response.

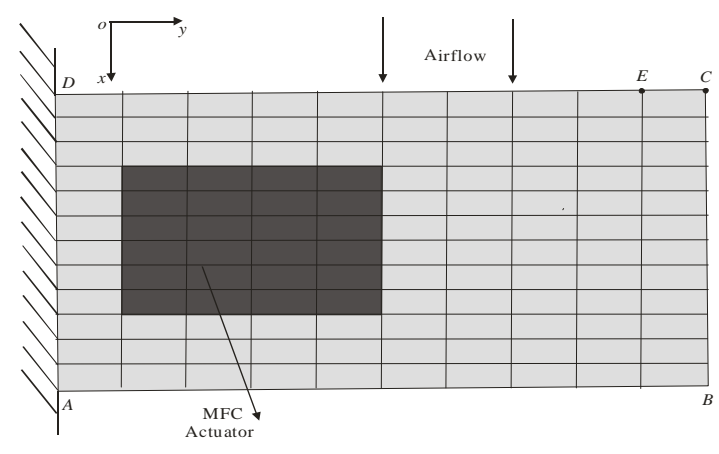

Fig.1 The model of cantilever plate with MFC

Table 1 The parameters of MFC

\begin{tabular}{cc}
\hline \multicolumn{2}{l}{ Piezolelctric strain } \\
constant & $d_{11}=4 \times 10^{-10}(\mathrm{C} / \mathrm{N}) \quad d_{12}=-1.7 \times 10^{-10}(\mathrm{C} / \mathrm{N})$ \\
\hline Elastic constant & $E_{1}=30.34(\mathrm{GPa}) \quad E_{2}=15.86(\mathrm{GPa}) \quad \mu_{12}=0.31$ \\
& $\mu_{21}=0.16 \quad \mu_{23}=0.31 \quad G_{12}=5.52(\mathrm{GPa})$ \\
Thickness & $0.3(\mathrm{~mm})$ \\
\hline
\end{tabular}

Table 3 Displacement RMS and percentage reduction

\begin{tabular}{cccc}
\hline Velocity $(\mathrm{m} / \mathrm{s})$ & $\bar{A}(\mathrm{~mm})$ & $\bar{B}(\mathrm{~mm})$ & $R(\%)$ \\
\hline 10 & 1.623 & 0.621 & 61.74 \\
20 & 1.350 & 0.576 & 57.33 \\
30 & 1.140 & 0.535 & 53.07 \\
40 & 0.974 & 0.501 & 48.56 \\
50 & 0.840 & 0.471 & 43.93 \\
60 & 0.726 & 0.439 & 39.53 \\
70 & 0.625 & 0.401 & 35.84 \\
80 & 0.533 & 0.367 & 31.14 \\
\hline
\end{tabular}

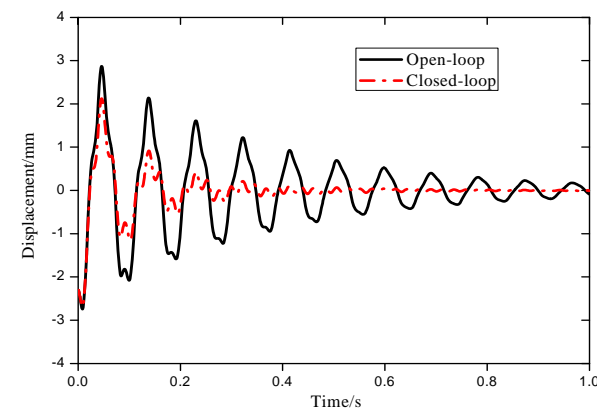

Fig.2 Displacement response of the aeroelasticity model

Table 2 The first five natural frequencies of the tail with MFC

\begin{tabular}{|c|c|c|c|c|c|}
\hline Mode & $\begin{array}{l}\text { The first } \\
\text { bending }\end{array}$ & $\begin{array}{l}\text { The first } \\
\text { rotation }\end{array}$ & $\begin{array}{l}\text { The second } \\
\text { bending }\end{array}$ & $\begin{array}{l}\text { The second } \\
\text { torsion }\end{array}$ & $\begin{array}{l}\text { The third } \\
\text { bending }\end{array}$ \\
\hline $\begin{array}{c}\text { Frequen } \\
(\mathrm{Hz})\end{array}$ & 10.89 & 48.77 & 67.26 & 155.3 & 187.1 \\
\hline
\end{tabular}

Table 4 Percentage of kinds of energy of the aeroelastic system under different airflow velocities

\begin{tabular}{cllll}
\hline Velocity $(\mathrm{m} / \mathrm{s})$ & $\bar{D}(\%)$ & $\bar{E}(\%)$ & $\bar{F}(\%)$ & $\bar{C}(\%)$ \\
\hline 10 & -8.94 & -45.57 & -54.51 & -11.71 \\
20 & -18.59 & -42.51 & -61.10 & -22.29 \\
30 & -28.29 & -39.64 & -67.93 & -32.36 \\
40 & -37.55 & -35.70 & -73.25 & -41.25 \\
50 & -46.83 & -30.98 & -77.81 & -48.97 \\
60 & -54.70 & -26.76 & -81.46 & -56.11 \\
70 & -62.36 & -21.99 & -84.35 & -65.67 \\
80 & -73.81 & -16.05 & -89.86 & -77.51 \\
\hline
\end{tabular}

It is calculated that the flutter velocity of the cantilever plate is $102.8 \mathrm{~m} / \mathrm{s}$, and the flutter frequency is $31.1 \mathrm{~Hz}$. At typical airflow velocity $50 \mathrm{~m} / \mathrm{s}$ below the flutter velocity, through applying active control, the closed-loop response of the aeroelastic model is significantly reduced compared with the open-loop response, as shown in Fig.2. Then the percentage reductions of displacements RMS at 1s of the output point are calculated under different airflow velocities below the flutter velocity, as shown in Fig.3. From the change of $\bar{A}$ in Table 3, the open-loop response of the aeroelastic model decreases little by little with the increase of the velocity. This shows that the additional aerodynamic forces play a role in damping of the aeroelastic open-loop system, and with the increase of airflow velocity, the damping effect on the system is more and more strong.

From the change of $R$ in Table 3, with the increase of airflow velocity, the percentage reductions of displacements RMS decrease gradually, that is, the active control effect of the aeroelastic model is little by little weak. This means that the damping effect of the piezoelectric driving force on the aeroelastic closed-loop system becomes gradually weak. Because with the increase of airflow 
velocity, the closed-loop displacement response decreases as shown as $\bar{B}$ in Table 3, the damping effect of the piezoelectric driving force on the aeroelasticity closed-loop system is increasingly strong.

Control mechanism analysis of the aeroelastic control model. Under the initial condition, the initial mechanical energy of the aeroelastic system is $3.4059 \times 10^{-3} \mathrm{~J}$. Then the system mechanical energy at a certain time is equal to the sum of the initial mechanical energy, the work done by the additional aerodynamic force and piezoelectric driving force at this time.

First of all, the ratio of the work done by the additional aerodynamic force to the initial mechanical energy at $0.1 \mathrm{~s}$ in the aeroelastic open-loop system under different airflow velocities, as shown as $\bar{C}$ in Table 4. In Table 4 the minus sign means that this force does negative work on the system. From the change of $\bar{C}$ in table 4, with the increase of airflow velocity, energy dissipation by the additional aerodynamic force becomes more and more. This means that in the open-loop system, the additional aerodynamic force plays a role in damping of the system, and this can also verify the conclusion mentioned above.

Afterwards, the ratio of the work by the additional aerodynamic force to the initial mechanical energy(namely, $\bar{D}$ ), the ratio of the work by the piezoelectric driving force to the initial mechanical energy(namely, $\bar{E}$ ) and the ratio of the total energy consumption to the initial mechanical energy(namely, $\bar{F}$ ) at 0.1 s in the aeroelastic closed-loop system are calculated respectively, which are respectively represented by $\bar{D}, \bar{E}$ and $\bar{F}$, as shown in Table 4, in order to explain the control mechanism in the aeroelastic control from the aspect of energy.

It can be seen from Table 4 that the piezoelectric driving force and the additional aerodynamic force all do negative work on the closed-loop system. They all consume the energy of the system and they are all a kind of damping force for the closed-loop system. Under the combined action of the piezoelectric driving force and the additional aerodynamic force, the vibration response of the aeroelastic system is gradually reduced, as shown in Fig.2. From the change of $\bar{E}$ in Table 4, below the flutter velocity, at lower airflow velocity, the piezoelectric driving force plays an important role in suppressing the vibration response. When the airflow speed is $0 \mathrm{~m} / \mathrm{s}$, the aeroelastic control model is changed into the structural vibration control model. At this time there is no additional aerodynamic force and the ratio of the work by the piezoelectric driving force to the initial mechanical energy is $-49.10 \%$. In the case of constant input voltage, with the increase of airflow velocity, the energy dissipation of the additional aerodynamic force is more and more, as shown as the change of $\bar{D}$ in Table 4, however, the energy consumption of the piezoelectric drive is less and less. That is to say, at middle and higher velocity below the flutter velocity, the additional aerodynamic force plays an important role in suppressing the vibration response. With the increase of airflow velocity, the total consumption energy of the system is increasing as shown as $\bar{F}$ in Table 4 , and correspondingly the system vibration response is becoming smaller and smaller.

In the active vibration control, the energy consumption of the vibration is completely dependent on the piezoelectric driving force; however in the aeroelastic active vibration control, the main role of the piezoelectric actuator is causing elastic deformation of the structure in the air flow, so that more additional aerodynamic forces are obtained due to the structural deformation. With the increase of airflow velocity, the effect of the piezoelectric driving force on suppressing vibration response becomes little by little weak, but the effect of the additional aerodynamic force gets more and more strong. The effects of the piezoelectric driving forces on the energy consumption between the vibration control system and the aeroelastic system are obviously different.

\section{Conclusions}

(1)Below the flutter velocity, the additional aerodynamic force is a kind of damping force for the aeroelastic open-loop system. 
(2)Below the flutter velocity, the piezoelectric driving force and the additional aerodynamic force are all a kind of damping force for the aeroelastic closed-loop system. At lower airflow velocity, the piezoelectric driving force plays an important role in suppressing the vibration response; but at middle and higher velocity below the flutter velocity, the additional aerodynamic force plays an important role in suppressing the vibration response.

(3)In the vibration control, the energy consumption of the vibration is completely dependent on the piezoelectric driving force; however, in the aeroelastic vibration control, it is mainly dependent on the additional aerodynamic force. There are significant differences between the mechanisms of the two control models.

\section{Acknowledgements}

This work was financially supported by the National Natural Science Foundation of China (11372320).

\section{References}

[1] S. Raja, A.A. Pashilkar, R. Sreedeep and J.V. Kamech: Aerospace Science and Technology Vol. 10(2006), p. 435-441

[2] J.-H. Han, J. Tani and J. Qiu: Journal of Sound and Vibration Vol. 291(2006), p. 706-722

[3] E.F. Sheta, R.W. Moses and L.J. Huttsell: Journal of Sound and Vibration Vol. 292(2006), p. 854-868

[4] X.J. Dong, G. Meng and J.C. Peng: Journal of Sound and Vibration Vol. 297(2006), p. 680-693

[5] M. Li, W.M. Chen, M.C. Wang, and L.J, Jia: Science in China Series E: Technological Science, Vol. 52(2009), p. 2576-2584

[6] E. Albano and W.P. Rodden: AIAA Journal Vol. 7(1969), p. 279-285

[7] J. Sun and M. Li: Engineering Mechanics Vol. 33(2016), p. 234-243(in Chinese) 